\title{
Inward electrostatic precipitation of interplanetary particles
}

\author{
Aaron J. Rulison \\ Mechanical Engineering, ${ }^{\text {a) }}$ California Institute of Technology, Pasadena, California 91125 \\ Richard C. Flagan \\ Chemical Engineering 210-41, California Institute of Technology, Pasadena, California 91125 \\ Thomas J. Ahrens \\ Lindhurst Laboratory of Experimental Geophysics, Seismological Laboratory 252-21, California Institute \\ of Technology, Pasadena, California 91125
}

(Received 29 March 1993; accepted for publication 3 March 1994)

\begin{abstract}
An inward precipitator collects particles initially dispersed in a gas throughout either a cylindrical or spherical chamber onto a small central planchet. The instrument is effective for particle diameters greater than about $1 \mu \mathrm{m}$. One use is the collection of interplanetary dust particles which are stopped in a noble gas (xenon) by drag and ablation after perforating the wall of a thin-walled spacecraft-mounted chamber. First, the particles are positively charged for several seconds by the corona production of positive xenon ions from inward facing needles placed on the chamber wall. Then an electric field causes the particles to migrate toward the center of the instrument and onto the planchet. The collection time (of the order of hours for a $1 \mathrm{~m}$ radius spherical chamber) is greatly reduced by the use of uptimally localed screens which reapportion the electric field. Some of the electric field lines terminate on the wires of the screens so a fraction of the total number of particles in the chamber is lost. The operation of the instrument is demonstrated by experiments which show the migration of carbon soot particles with radius of approximately $1 \mu \mathrm{m}$ in a 5-cm-diam cylindrical chamber with a single field enhancing screen toward a $3.2 \mathrm{~mm}$ central collection rod.
\end{abstract}

\section{INTRODUCTION}

Orbits of interplanetary dust particles (IDPs) can be used to constrain their origins, e.g., asteroidal, cometary, or interstellar. IDPs in elliptic orbits are thought to originate from asteroidal collisions, while those in highly eccentric or inclined orbits are probably associated with comets. IDPs in hyperbolic orbits may originate in interstellar space. If the orbits of individual IDPs could be determined, analysis of those particles would provide important insights into the compositions of objects in the various regions of space. ${ }^{1,2}$

Collection of interplanetary dust particles requires a large cross-section sampler to achieve reasonable encounter rates, while analysis of the collected particles requires that they be precisely located. A number of systems satisfy the former requirement. Notably, particle collection in aerogels can provide samples for earth-based analyses. ${ }^{3}$ In-flight analysis is complicated by the difficulty of locating particles within the aerogel. In this paper we propose collecting the particles in a dense gas contained in a thin-walled vessel. ${ }^{4}$ The collector illustrated in Fig. 1 collects particles impinging from a limited range of solid angles, and then transports the particles to planchets located on a carousel at the center of the associated spherical segment of the collector. Particle orbits can be identified from luminous trails that will be recorded using video cameras as the particles undergo ablative deceleration in the gas.

Once stopped by the gas drag, the particles are mobile and can be charged and then directed onto a small planchet for subsequent analysis. In typical applications of electro-

\footnotetext{
"Now at the Jet Propulsion Laboratory 183-401, 4800 Oak Grove Drive, Pasadena, CA 91109.
}

static precipitation for particle collection, ions produced by a corona discharge from a central wire attach to particles that then drift under electrostatic forces toward large area collection plates. Because of the large collection area, the times required to coat the electrodes with particles are relatively long. This is useful in gas cleaning applications, but would be unacceptable in the sampling of particles for microscopy or other analysis due to the low density of deposits that would result. This difficulty is overcome by inverting the system: ions are generated near the surface of the large area electrode and drift toward a small collector. Since the directions from which IDPs will enter the collector are not known, it is natural to place the planchet at the center of a spherical collector (the sampler illustrated in Fig. 1 would collect particles from a limited solid angle with the planchet mounted on a carousel at the center of the associated spherical segment).

The inward precipitator of Fig. 2(a) has a small spherical planchet at the center of a spherical cavity. (The following discussion also applics to an inward precipitator utilizing a small cylindrical planchet at the center of a cylindrical cavity.) The particle enters the chamber at hypersonic velocities and is decelerated. This is represented by the dashed segment of the trajectories shown in Fig. 2. After the particle is stopped, numerous corona needles placed on the wall act to charge the IDPs positively by producing a flood of positive ions inside the chamber when an electrical potential of about $+20 \mathrm{kV}$ is applied for several seconds. Positive ions are used since xenon is a poor absorber of electrons and, thus, would be ineffective for particle charging if used with a negative corona. An electric field $E$ is oriented to drive the particles to the planchet after they are charged. This is represented by the dotted segment of the trajectories shown in Fig. 2. The ge- 


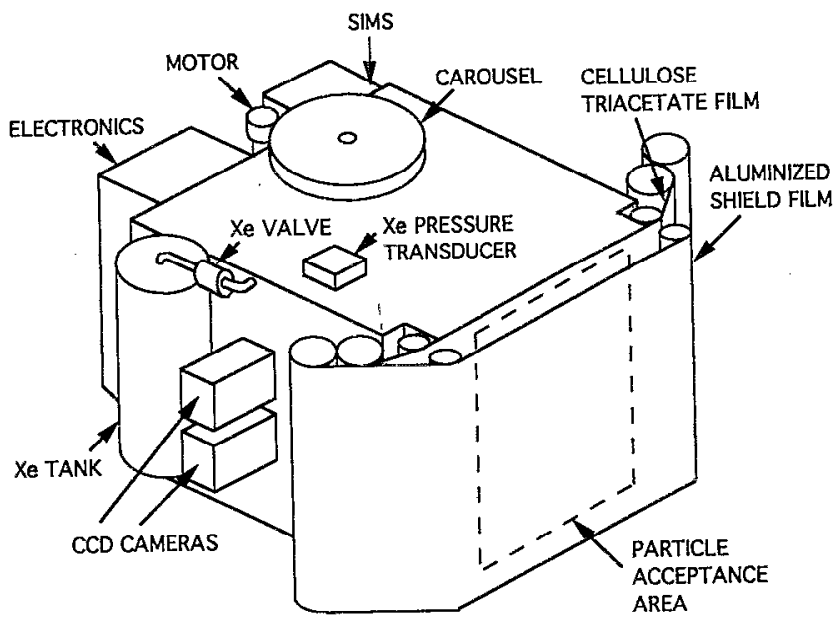

FIG. 1. Concept drawing for the dust and ring analyzer.

ometry of Fig. 2(a) has limitations in that $E$ is weak in regions far from the planchet and the migration speed of the particle is low there, resulting in long collection times $(\sim 1000 \mathrm{~h}$ for $5 \mu \mathrm{m}$ particles in a $1 \mathrm{~m}$ radius spherical chamber). To increase the field strength far from the planchet, one or more intermediate reapportioning screens are added [Fig. 2(b)]. As will be shown below, the screens significantly enhance particle migration and reduce the collection time.

This paper presents the techniques used for particle charging and a detailed analysis needed to determine the positions of the reapportioning screens that minimize the collection time in both the cylindrical and spherical gcometries. Results of some preliminary experiments with a working cylindrical model are presented and compared with theoretical performance.

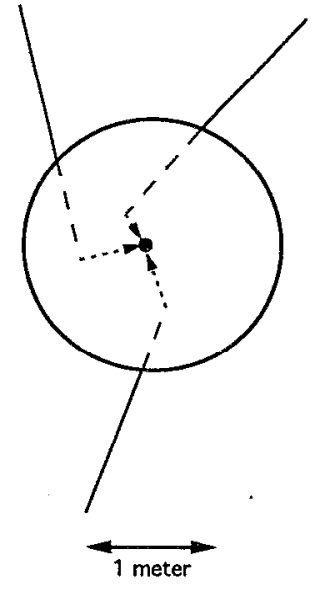

(a)

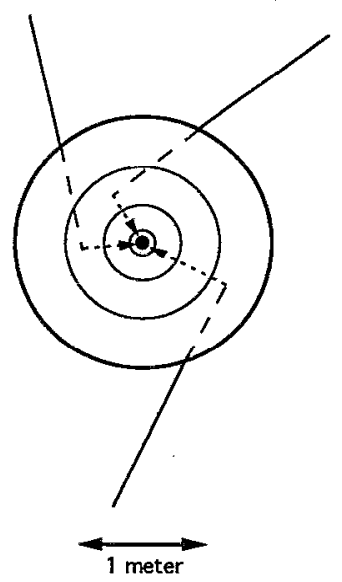

(b)
FIG. 2. Geometry of the inward clectrostatic precipitator. Particles dispersed throughout the chamber after being stopped by gas drag and ablation are collected by charging them and applying an electric field to force them toward the small planchet at the center. Solid lines indicate trajectories (relative to the spacecraft) of IDPs in space. Dashed lines indicate trajectories during deceleration. Dotted lines indicate trajectories during electrostatic precipitation. Three electric field reapportioning screens are shown in part (b).

\section{THEORY}

In the analysis of the particle' migration we will assume that the particle is spherical and homogeneous. Although IDPs are known to have complex shapes and structures (see, e.g., Ref. 5), little is known about the morphological change accompanying penetration into and ablation in the xenon filled chamber. The following analysis can be extended to account for nonspherical shapes and inhomogeneous internal structures when such data become available.

\section{A. Particle charging in a gas}

A micron-size dielectric particle suspended in a gas can be charged with tens or hundreds of elementary charges using corona field charging techniques. At the end of a sharp needle that is held at a positive potential, the electric field lines are directed outward, and the small numbers of free electrons that are present in the gas are accelerated toward the needle. If the potential is high enough then these electrons will be accelerated to sufficiently high kinetic energies $(\sim 12 \mathrm{eV})$ to ionize xenon atoms. Each collision liberates additional electrons and positive xenon ions, establishing a positive corona around the needle tip. The positive xenon ions are repelled from the needle and out into the surrounding gas. It is these positive ions which attach to and charge the IDPs in the gas.

It is also possible to operate negative coronas by applying a negative potential to the needle, but for particle charging applications this requires a surrounding gas that is an efficient absorber of electrons. Since noble gases are not efficient absorbers of electrons due to their low electron affinities, ${ }^{6}$ the negative corona is not used.

Field charging occurs when the positive ions created by the corona migrate along electric field lines and impinge on the particle. As the charge on the particle increases the electric field in the vicinity is altered. At high particle charge levels, incoming positive ions are deflected, and field charging ceases at the saturation field charge $q_{s}$. The time required to reach $q_{s}$ is ${ }^{7}$

$$
\tau_{c}=\frac{4 \epsilon_{0}}{e B_{i} N_{i_{\infty}}}
$$

where $\tau_{c}$ is the time for complete field charging, $\epsilon_{0}$ is the permittivity of free space, $e$ is the elementary charge, $B_{i}$ is the ion mobility, and $N_{i_{\infty}}$ is the background ion number concentration. For the operating conditions of the proposed collector (xenon gas at $0.2 \mathrm{~atm}$ and $270 \mathrm{~K}$ ), Eq. (1) gives $\tau_{c} \sim 0.1 \mathrm{~s}$. This time is much less than the overall collection times in a chamber of the size being considered (which is of the order of $1 \mathrm{~m}$ in radius) so the particles can be assumed to achieve the saturation field charge rapidly. The saturation charge is ${ }^{7}$

$$
q_{s}=4 \pi \epsilon_{0} \frac{3 \kappa}{\kappa+2} E_{\infty} R_{p}^{2}
$$

where $\kappa$ is the dielectric constant of the particle, $E_{\infty}$ is the magnitude of the background electric field, and $R_{p}$ is the particle radius. Equation (2) is derived assuming a spherical and homogeneous particle. Typical values of $\kappa$ for mineral 
particles as might be encountered in interplanetary space range from approximately 2 to 10 . Values for metallic particles would be higher.

As long as the background xenon ions are present, ions continue diffusing to the particle surface, thereby increasing its charge beyond the value given by Eq. (2). ${ }^{7}$ It is assumed here, however, that potentials high enough to cause electrical discharges at the corona needles will be applied only long enough (several seconds) to reach the field charging saturation charge $q_{s}$. The ion migration time through a $1 \mathrm{~m}$ radius chamber with three screens is about $3 \mathrm{~s}$, assuming an ion mobility of $10^{-4} \mathrm{~m}^{2} \mathrm{~V}^{-1} \mathrm{~s}^{-1}$ and $V_{T}=20 \mathrm{kV}$. Hence, the ions will quickly be removed from the chamber, and the final particle charge will be $q_{s}$.

\section{B. Particle motion}

\section{Spherical case}

In order to describe the migration of a particle in the spherical chamber the electric field must be known throughout. Assuming the space charge density to be negligible, the potential between two concentric spheres is given by Laplace's equation in spherical coordinates with radial dependence only,

$$
\frac{1}{r^{2}} \frac{\partial}{\partial r}\left(r^{2} \frac{\partial V}{\partial r}\right)=0 \text {. }
$$

For $r_{i}>r_{i}$ where $r_{i}$ and $r_{j}$ are the radii of the spheres which are held at potentials $V_{i}$ and $V_{j}$, respectively, the solution to Eq. (3) in the region between the concentric spheres is

$$
V(r)=\frac{1}{r_{j}-r_{i}}\left(\frac{r_{i} r_{j}}{r}\left(V_{i}-V_{j}\right)+r_{j} V_{j}-r_{i} V_{i}\right)
$$

with $r_{i} \leqslant r \leqslant r_{j}$. The corresponding radial electric field strength is

$$
E(r)=-\frac{\partial V}{\partial r}=\frac{1}{r^{2}}\left(\frac{V_{i}-V_{j}}{r_{j}-r_{i}}\right) r_{i} r_{j} .
$$

The particles, having been charged positively to the field charge saturation level, will migrate toward the sphere center under the action of the electric field. For Stokesian particles (those with Reynolds numbers $\operatorname{Re}=2 u_{p} R_{p} \rho / \mu<0.1$, where $u_{p}$ is the particle speed, $R_{p}$ is the particle radius, $\rho$ is the gas density, and $\mu$ is the absolute viscosity) the radial migration velocity speed is ${ }^{7}$

$$
u_{r}=\frac{q_{s} E_{r}}{6 \pi \mu R_{p}},
$$

where $r$ is the radial position of the particle. Equation (6) is valid for approximately $1 / 2<R_{p}<5 \mu \mathrm{m}$. The lower limit is set by the requirement that the particle is much larger than the mean free path (which is about $0.3 \mu \mathrm{m}$ for xenon at 0.2 atm and $300 \mathrm{~K}$ ) and the upper limit is set by the Stokes flow regime (low $\mathrm{Re}$ ) requirement. For particles outside this range, both Eqs. (2) and (6) would need to be modified.

Rearranging and integrating Eq. (6) gives

$$
\int_{r_{i}}^{r_{j}} \frac{6 \pi \mu R_{p}}{q_{s} E} d r=\int_{t_{i}}^{t_{j}} d t=t_{j}-t_{i} .
$$

To find the time $T=t_{i}-t_{j}$ for the particle to move from one screen to the next in the spherical chamber, we set $r_{i}$ and $r_{j}$ to the radial locations of the screens. Substituting Eq. (5) gives

$$
T=\int_{r_{i}}^{r_{j}}\left(6 \pi \mu R_{p} d r\right) /\left(\frac{q_{s}}{r^{2}} \frac{V_{i}-V_{j}}{r_{j}-r_{i}} r_{i} r_{j}\right) .
$$

Since the particle is positively charged $\left(q_{s}>0\right)$, we must have $V_{i}<V_{j}$ to move the particle toward the sphere center. Thus

$$
\begin{aligned}
T & =\frac{6 \pi \mu R_{p}}{r_{i} r_{j} q} \frac{r_{j}-r_{i}}{V_{j}-V_{i}} \int_{r_{i}}^{r_{j}} r^{2} d r \\
& =\frac{2 \pi \mu R_{p}}{q} \frac{r_{j}-r_{i}}{V_{j}-V_{i}} \frac{r_{j}^{3}-r_{i}^{3}}{r_{i} r_{j}}>0 .
\end{aligned}
$$

The total time $T_{T}$ for the particle to pass from the outside of a spherical chamber with $n$ internal screens to the central planchet is a sum over the $n+1$ zones between the screens. Thus,

$$
T_{T}=\frac{2 \pi \mu R_{p}}{q_{s}} \sum_{i=0}^{n} \frac{r_{i+1}-r_{i}}{V_{i+1}-V_{i}} \frac{r_{i+1}^{3}-r_{i}^{3}}{r_{i+1} r_{i}},
$$

where $r_{n+1}=R$ is the overall radius and $r_{0}$ is the planchet radius.

One way to set the potentials on each screen is to divide the total available potential $V_{T}$ into even portions, so that $V_{j}-V_{i}=$ const $=V_{T} /(n+1)$. Thus $V_{i}=(i-1) V_{T} /(n+1)$. By fixing the voltages on the screens, the number of independent variables in the system available for minimizing $T_{T}$ is reduced from $2 n$ to $n$. The minimum in $T_{T}$ that is found will not be the absolute optimum, but the beneficial effect of the screens is still obvious and the calculation may be extended to include varying voltages in a straightforward manner. An example of this is given later. For now we have $V_{i+1}-V_{i}=\Delta V=V_{T} /(n+1)$ and Eq. (10) becomes

$$
T_{T}=\frac{2 \pi \mu R_{p}(n+1)}{q_{s} V_{T}} \sum_{i=0}^{n} \frac{\left(r_{i+1}-r_{i}\right)\left(r_{i+1}^{3}-r_{i}^{3}\right)}{r_{i+1} r_{i}} .
$$

If we now define the dimensionless radial coordinate $\hat{r}=r / R$ and the dimensionless time

$$
\hat{T}_{T}=T_{T} /\left(\frac{2 \pi \mu R_{p} R^{2}}{q_{s} V_{T}}\right)
$$

we obtain

$$
\hat{T}_{T}=(n+1) \sum_{i=0}^{n} \frac{\left(\hat{r}_{i+1}-\hat{r}_{i}\right)\left(\hat{r}_{i+1}^{3}-\hat{r}_{i}^{3}\right)}{\hat{r}_{i+1} \hat{r}_{i}} .
$$

Proper positioning of the screens minimizes $\hat{T}_{T}$. The number of independent variables available for minimizing $\hat{T}_{T}$ is $n$, namely the screen positions $\hat{r}_{1}, \ldots, \hat{r}_{n}$, with the restriction

$$
1>\hat{r}_{n}>\hat{r}_{n-1}>\hat{r}_{n-2}>\cdots>\hat{r}_{1}>\hat{r}_{p} \text {. }
$$

The total time $\hat{T}_{T}$ in Eq. (13) was minimized numerically for $n=1,2$, and 3 and for $\hat{r}_{p}=0.001$. This was done by systematically moving the screens throughout all of their al- 
TABLE I. Optimal screen positions for a spherical inward electrostatic precipitator

\begin{tabular}{ccc}
\hline \hline$\hat{T}_{r}$ & Number of screens & Screen positions $(\hat{r})$ \\
\hline 999 & 0 & $\ldots$ \\
17.7 & 1 & 0.135 \\
7.47 & 2 & $0.069,0.406$ \\
5.44 & 3 & $0.049,0.254,0.595$ \\
\hline
\end{tabular}

lowed positions and noting the positions corresponding to minimum $\hat{T}_{T}$. Table I shows the results. For a discussion of numerical analysis techniques for performing global optimization, see Press et al. ${ }^{8}$

By the addition of a single screen the collection time is reduced to $1.8 \%$ of its original value. Diminishing returns are seen with additional screens but with three screens the collection time is reduced to $0.54 \%$ of its original value. The number of screens to be used in the actual design will depend on trade-offs of collection time versus instrument mass, etc. The dimensionless electric fields $E /(V / R)$ vs $\hat{r}$ without screens and with three screens are shown in Fig. 3. The screens add electric field strength to the outer regions of the chamber where the migration velocities would otherwise be very low. The dimensionless particle motion characteristics for these two geometries are shown in Fig. 4 where $\hat{t}=t q_{s} V_{T} /\left(2 \pi \mu R_{p} R^{2}\right)$ is the dimensionless time. The screens significantly reduce the time required to bring the sample to the planchet at $\hat{r}=0.001$. For a concrete example, consider particles in a $1 \mathrm{~m}$ radius chamber with three screens filled with xenon gas at $300 \mathrm{~K}$ and $0.2 \mathrm{~atm}$ and with $+20 \mathrm{kV}$ total available potential. The potentials are thus $+20,+15$, $+10,+5$, and $0 \mathrm{kV}$ on the outer wall, screens, and planchet, respectively. Collection times are shown as a function of particle size and dielectric constant in Fig. 5. The collection time is inversely proportional to the particle size and dielectric constant.

The dimensionless size of the planchet has an effect on

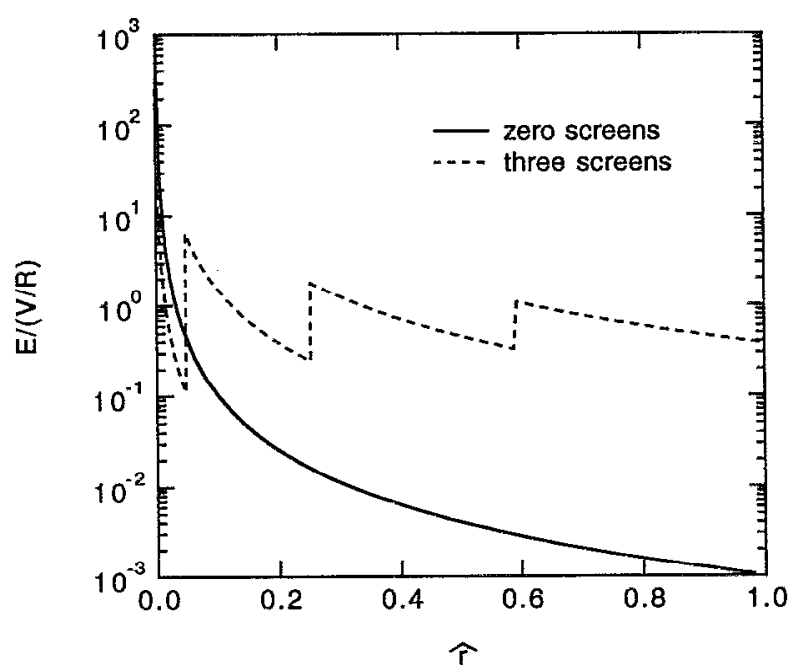

FIG. 3. Dimensionless electric field strength vs dimensionless radial position for spheres with zero screens and three optimally positioned screens. The screens increase the field strength in the outer part of the chamber.

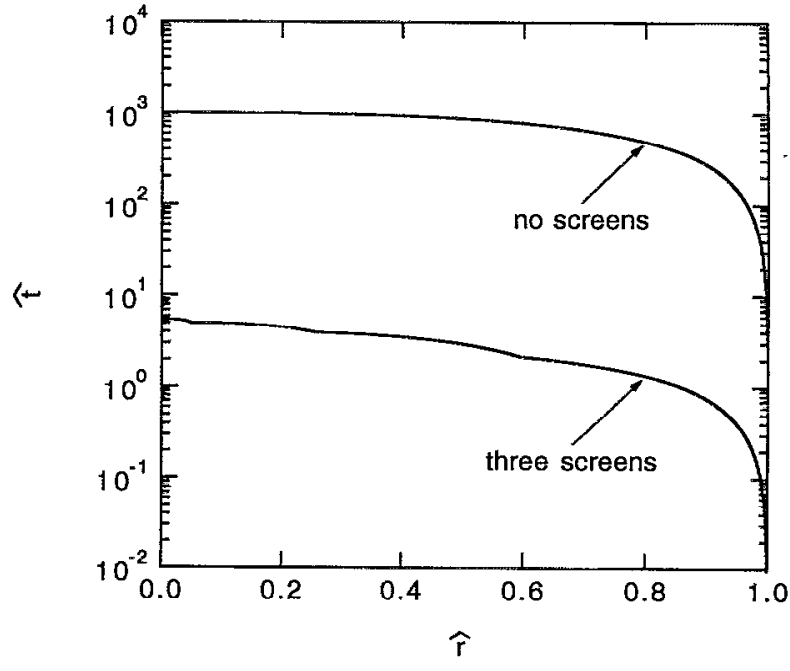

FIG. 4. Dimensionless particle motion characteristics for a sphere with zero and three screens. The screens significantly decrease the dimensionless time required to bring the sample to the planchet at $\hat{r}=0.001$.

the collection times and screen positions. For planchets with radii of $0.05 \%$ and $0.2 \%$ of the overall sphere radius, the dimensionless collection times for three screens are 5.75 and 5.11, respectively. Also, as given in Table II the optimum screen positions are such that the screens are placed closer to the planchet for the smaller planchet.

If we relax the restriction that the potential is equally divided over the zones of the chamber then $\vec{T}_{T}$ can be further reduced. For example, consider a spherical chamber with one internal screen $(n=1)$ and $\hat{r}_{p}=0.001$. Both the screen position and potential are allowed to vary such that $\hat{T}_{T}$ is a function of $\hat{r}_{2}$ and $\hat{V}_{2}$, where $\hat{r}_{1}<\vec{r}_{2}<1$ and $0<V_{2}<V_{T}$. For this case, $\hat{T}_{T}=15.9$ with $\hat{r}_{2}=0.106$ and $V_{2}=0.272 V_{T}$. This compares to the one screen case in Table $\mathrm{I}$, which has $\hat{T}_{T}=17.7$, $\hat{r}_{2}=0.135$, and $V_{2}=0.500 V_{T}$. Thus a reduction in $\hat{T}_{T}$ is ob-

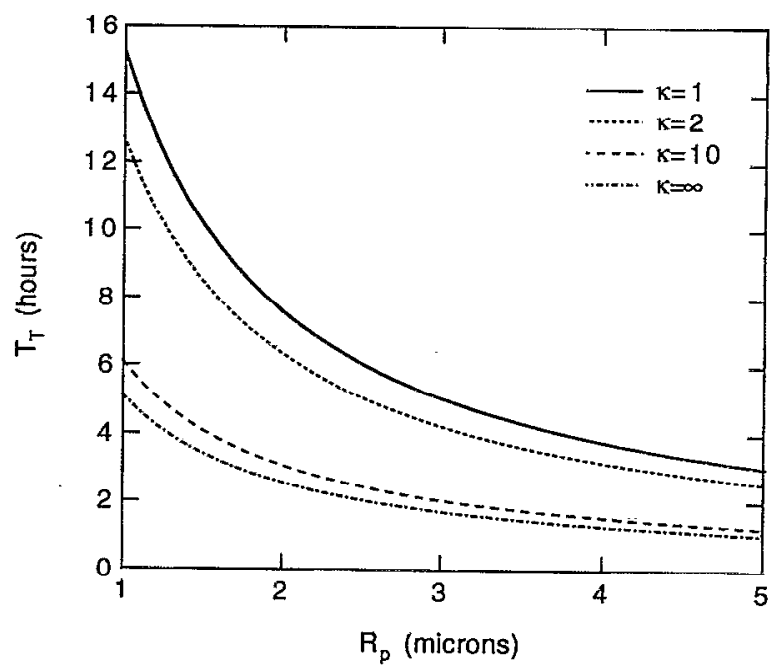

FIG. 5. Collection time as a function of particle diameter and dielectric constant. Large particles with large dielectric constant are collected in the least amount of time $\left(\mu=5 \times 10^{-5} \mathrm{~kg} \mathrm{~m}^{-1} \mathrm{~s}^{-1}, E_{\infty}=1.4 \times 10^{4} \mathrm{~V} / \mathrm{m}, R=1 \mathrm{~m}\right.$, $V_{r}=20 \mathrm{kV}$, and $\left.\hat{T}_{T}=5.44\right)$. 
TABLE II. Effect of dimensionless planchet size.

\begin{tabular}{ccc}
\hline \hline$\hat{T}_{T}$ & Number of screens $(n)$ & Screen positions $(\hat{r})$ \\
\hline $\begin{array}{c}\text { For } \hat{r}_{p}=0.0005: \\
1999 \\
5.75\end{array}$ & 0 & $\ldots$ \\
For $\hat{r}_{p}=0.002:$ & 3 & $0.038,0.232,0.579$ \\
499 & & \\
5.11 & 0 & $\ldots .062,0.277,0.612$ \\
\end{tabular}

tained by reducing the potential on the screen and moving it slightly inward toward the planchet.

\section{Cylindrical case}

The cylindrical geometry can also be treated using the above analysis. The governing equation for the potential is

$$
\frac{1}{r} \frac{\partial}{\partial r}\left(r \frac{\partial V}{\partial r}\right)=0
$$

with $V=V_{i}$ at $r=r_{i}, V=V_{j}$ at $r=r_{j}$, where $r_{i}$ and $r_{j}$ are the radii of cylinders which are held at potentials $V_{i}$ and $V_{j}$, respectively. The solution to Eq. (15) is

$$
V(r)=\frac{V_{j} \ln \left(r / r_{j}\right)-V_{i} \ln \left(r / r_{j}\right)}{\ln \left(r_{j} / r_{i}\right)}
$$

for $r_{i}<r<r_{j}$. The electric field is

$$
E(r)=\frac{V_{i}-V_{j}}{r \ln \left(r_{j} / r_{i}\right)} .
$$

Equation (7) then gives

$$
t_{i}-t_{j}=\int_{r_{i}}^{r_{j}}\left\{\left(6 \pi \mu R_{p}\right) /\left[\frac{q\left(V_{i}-V_{j}\right)}{r \ln \left(r_{j} / r_{i}\right)}\right]\right\} d r .
$$

Assuming $q>0$ and $V_{j}>V_{i}$ gives

$$
\begin{aligned}
t_{i}-t_{j} & =\frac{6 \pi \mu R_{p} \ln \left(r_{j} / r_{i}\right)}{q\left(V_{j}-V_{i}\right)} \int_{r_{i}}^{r_{j}} r d r \\
& =\frac{3 \pi \mu R_{p} \ln \left(r_{j} / r_{i}\right)}{q\left(V_{j}-V_{i}\right)}\left(r_{j}^{2}-r_{i}^{2}\right)>0 .
\end{aligned}
$$

The total collection time for the cylindrical chamber with $n$ internal screens is

$$
T_{T}=\underset{q}{3 \pi \mu r_{p}} \sum_{i=0}^{n} \frac{\ln \left(r_{j} / r_{i}\right)}{V_{j}-V_{i}}\left(r_{j}^{2}-r_{i}^{2}\right),
$$

where $r_{n+1}=R$ and $r_{0}=r_{p}$. If we set $V_{j}-V_{i}=$ const, then

$$
T_{T}=\frac{3 \pi \mu R_{p}(n+1)}{q V_{T}} \sum_{i=0}^{n} \ln \left(r_{j} / r_{i}\right)\left(r_{j}^{2}-r_{i}^{2}\right) .
$$

Using the previously defined dimensionless variables we obtain

$$
\hat{T}_{T}=\frac{3}{2}(n+1) \sum_{i=0}^{n} \ln \left(\hat{r}_{j} / \hat{r}_{i}\right)\left(\hat{r}_{j}^{2}-\hat{r}_{i}^{2}\right) .
$$

Table III shows the results of minimizing $\hat{T}_{T}$ for the cylin-
TABLE III. Optimal screen positions for a cylindrical inward electrostatic precipitator.

\begin{tabular}{ccc}
\hline \hline$\hat{T}_{T}$ & Number of screens $(n)$ & Screen positions $(\hat{r})$ \\
\hline 10.4 & 0 & $\ldots$ \\
4.83 & 1 & 0.301 \\
3.98 & 2 & $0.179,0.569$ \\
3.66 & 3 & $0.130,0.400,0.697$ \\
\hline \hline
\end{tabular}

drical case for one, two, and three screens for $\hat{r}_{p}=0.001$.

A cylinder collects a particle in a shorter time than a sphere of the same radius that is held at the same potential (Table I). For example, with three screens the collection time for a cylindrical chamber is $67 \%$ that of a spherical one. The screens enhance the collection speed, although the increase is not as great as for the spherical case because the electric field in the cylindrical gcomctry drops off Icss rapidly away from the planchet, and reapportioning the electric field has less benefit. One screen reduces the collection time to $46.4 \%$ of the value for zero screens while three screens reduce it to $35.2 \%$. The influence of the dimensionless planchet size and variable voltages is qualitatively similar to that for the spherical geometry.

\section{Particle loss to the screens}

Since a fraction of the electric field lines around the screen wires terminate on the wires, some particle losses will occur as particles migrate across the chamber. The magnitude of this loss depends on the details of the screen mesh size and shape and wire size and shape. It also depends on the physical characteristics of the particles since there are aerodynamic and inertial forces involved in the migration. To illustrate how loss can occur, we considered an infinite array of wires between two parallel plates. The upper plate (at $\hat{y}=1$ ) is held at a potential $\hat{V}_{3}=1$ (where $\hat{V}=V / V_{T}$ ) and the lower plate (at $\hat{y}=0$ ) is grounded. The wires (at $\hat{y}=y_{2}$ ) are held at a potential $\hat{V}_{2}$ where $\hat{V}_{1}<\hat{V}_{2}<\hat{V}_{3}$. A positively charged particle would thus migrate from the top plate toward the bottom plate. In both the cylindrical and spherical geometries considered above the screens strengthen the electric field in the outer part of the chamber and weaken it in the inner part. This can be simulated in the test geometry by setting, for example, $\hat{V}_{2}=0.5$ and $\hat{y}_{2}>0.5$. To obtain the field lines the potential field $\hat{V}(\hat{x}, \hat{y})$ must be solved. The governing equation, assuming no space charge, is the Laplace equation in rectangular coordinates

$$
\frac{\partial^{2} \hat{V}}{\partial \hat{x}^{2}}+\frac{\partial^{2} \hat{V}}{\partial \hat{y}^{2}}=0
$$

The boundary conditions for Eq. (23) are $\hat{V}$ (wire) $=\hat{V}_{2}$, $\hat{V}(\hat{y}=1)=1, \quad \hat{V}(y=0)=0$, and $(\partial \hat{V} / \partial \hat{x})(\hat{x}=0, \hat{x}=1)=0$. Equation (23) was solved numerically using a finite-element method. ${ }^{9}$ The wire surface is simulated by setting four points in the numerical grid equal to $\hat{V}_{2}$. The resulting field lines are shown in Fig. 6. In this example $40 \%$ of particles beginning at random $\hat{x}$ locations along $\hat{y}=1$ migrate along field lines which terminate on the wire. These particles will eventually impact the wire and be lost. The remaining particles 


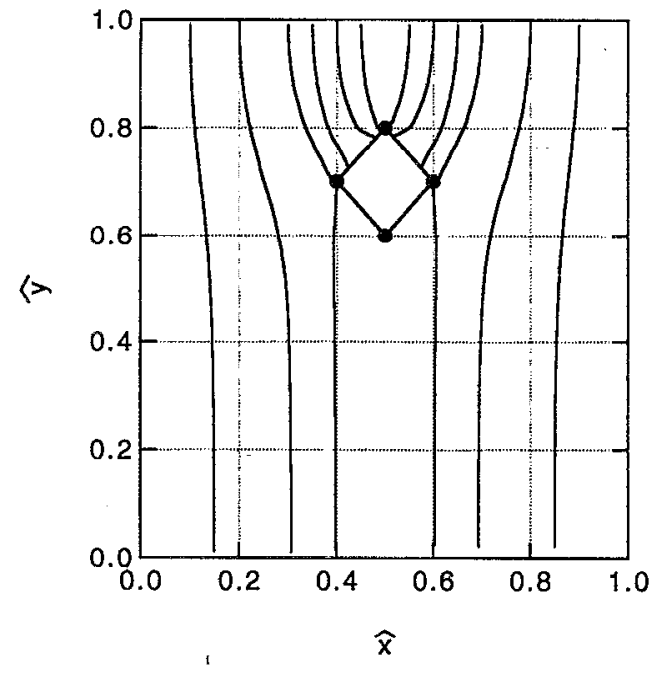

FIG. 6. Field lines according to the finite element solution. One section of the periodic geometry that extends to infinity to the left and right is shown.

will continue to migrate towards the central collectors. In the actual instrument an experimental calibration or full threedimensional simulation is needed to quantify the loss.

\section{EXPERIMENTS}

To demonstrate the operation of the inward electrostatic precipitator, a cylindrical chamber was constructed (Fig. 7). A cylindrical geometry was chosen for ease of experiment and construction. The brass outer casing was $5.1 \mathrm{~cm}$ diam. The ends and sides were fitted with clear Lucite ports to allow videotaping and $\mathrm{He}-\mathrm{Ne}$ laser illumination of the particles. The particles were injected using a syringe through a port in the side of the casing and allowed to diffuse throughout the interior of the chamber prior to applying any potentials. A long range microscope (Questar QM1), CCD camera (COHU 4815-5000/0000), and video cassette recorder ( $\mathrm{Pa}-$ nasonic AG-1830 in S-VHS format) were used to record the

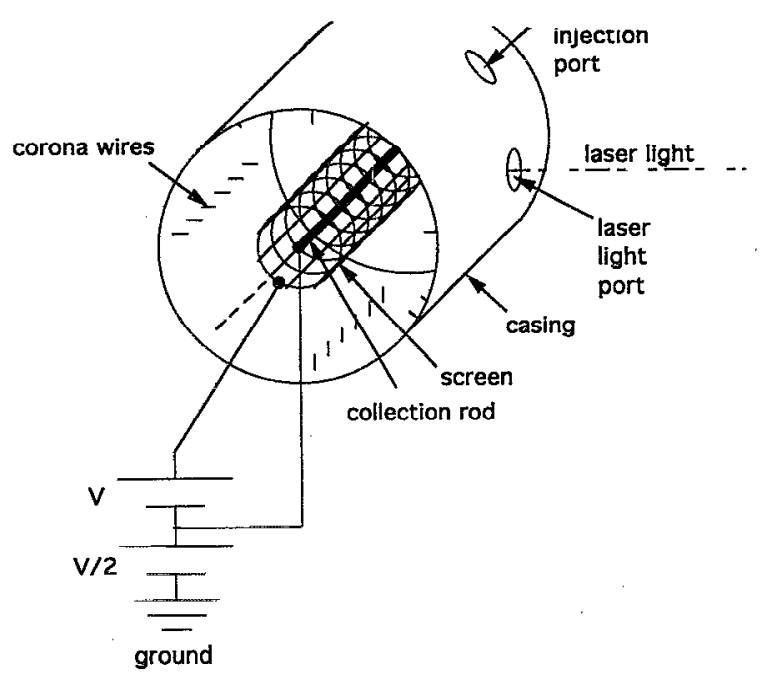

FIG. 7. Schematic drawing of the cylindrical inward precipitator constructed for the experiments.
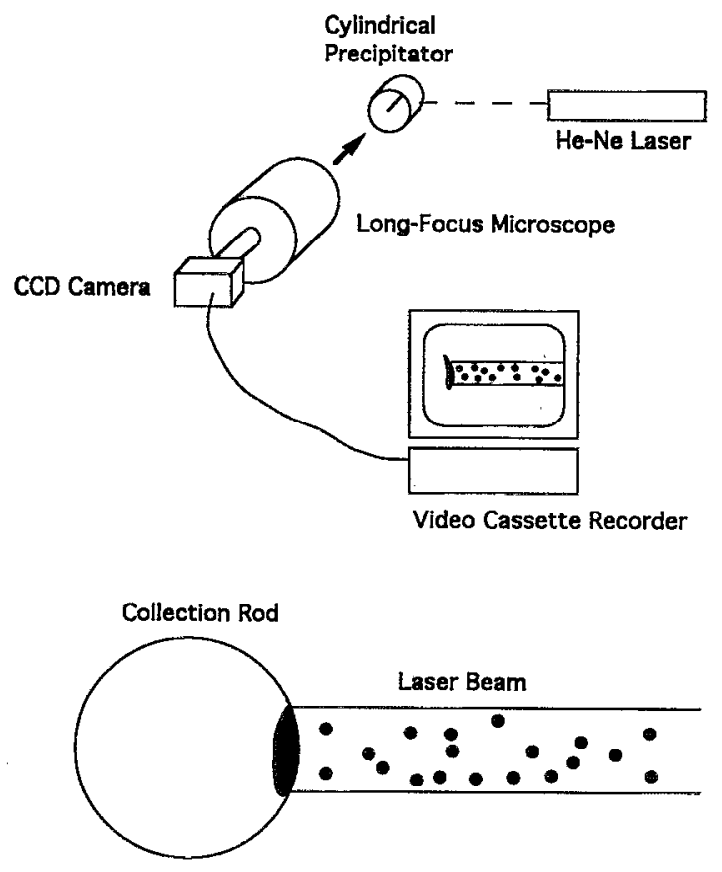

FIG. 8. Overall experimental apparatus. The particle motion was recorded using a long range microscope and video cassette recorder.

motion of the particles (Fig. 8). The particles wcre charged by 1-2 $\mathrm{s}$ of corona discharge of positive ions from corona needles held at $+10 \mathrm{kV}$ and placed at the perimeter of the precipitator. After charging, the potential was reduced to +6 $\mathrm{kV}$ to stop the corona but maintain an electric field. The particles then migrated toward the $3.2-\mathrm{mm}$-diam grounded collection rod. A single $2.5-\mathrm{cm}$-diam screen electrode was held at half the casing potential (i.e., $+5 \mathrm{kV}$ during charging and $+3 \mathrm{kV}$ during collection).

The particles were carbon soot produced from a candle flame. ${ }^{10}$ To obtain the particles, a glass container was placed over the flame until it extinguished, producing large amounts of the soot aerosol. The particles were extracted from the container with the syringe and then injected into the cylindrical precipitator. Some large-scale motion occurred just after injection into the chamber but this ceased after approximately $1 \mathrm{~min}$. The appearance of particles in the chamber before any potential was applied is shown in Fig. 9 which is a photograph taken directly from a black and white monitor. On the original video recording, the particles were seen to move under Brownian motion and slow gravitational settling, but all large-scale fluid motion had ceased. Note that the images of particles formed by the scattered laser light are not indicative of the actual particle size due to insufficient camera resolution.

After charging, all particles migrated toward the collection rod. The speed varied from particle to particle due to the variation of size and charge. Figure 10 shows photographs of a particularly slow particle taken at $0.17 \mathrm{~s}$ intervals. The average speed was $4.5 \mathrm{~mm} / \mathrm{s}$. This particle was chosen for photographing since its slow speed facilitated better images for the figures in this paper.

The experimentally determined speed cannot be precisely compared with the theoretical predictions since the 


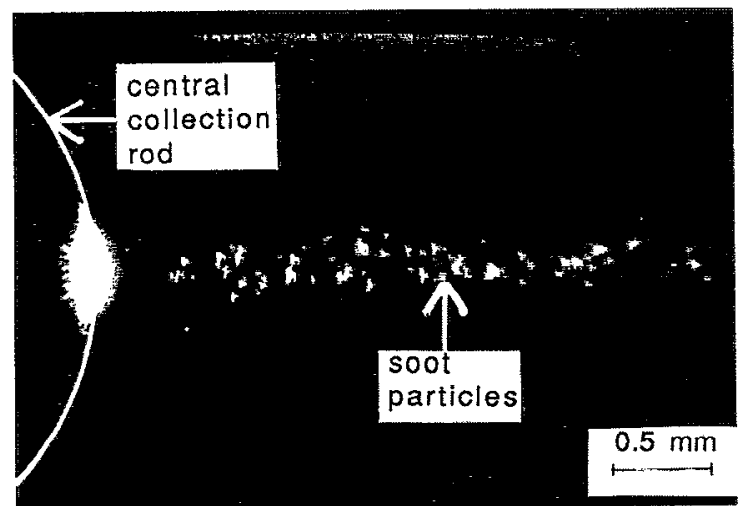

FIG. 9. Photograph of particles near the central collection rod (end view). The outline of the collection rod is drawn. Scattered light from the laser is seen at the surface of the rod. No charge or electric field exist. The size of a particle image does not represent the actual particle size due to insufficient camera resolution.

exact size and charge of the particle seen in the videotape was not known. By the observed settling speed of the particles in the chamber, the average particle diameter was roughly $1 \mu \mathrm{m}$. Using Eq. (2) for the charge in the migration velocity calculation [Eq. (6)] gives $43 \mathrm{~mm} / \mathrm{s}$, which is one order of magnitude higher than observed. The particle in Fig. 10 probably did not receive the full saturation charge given
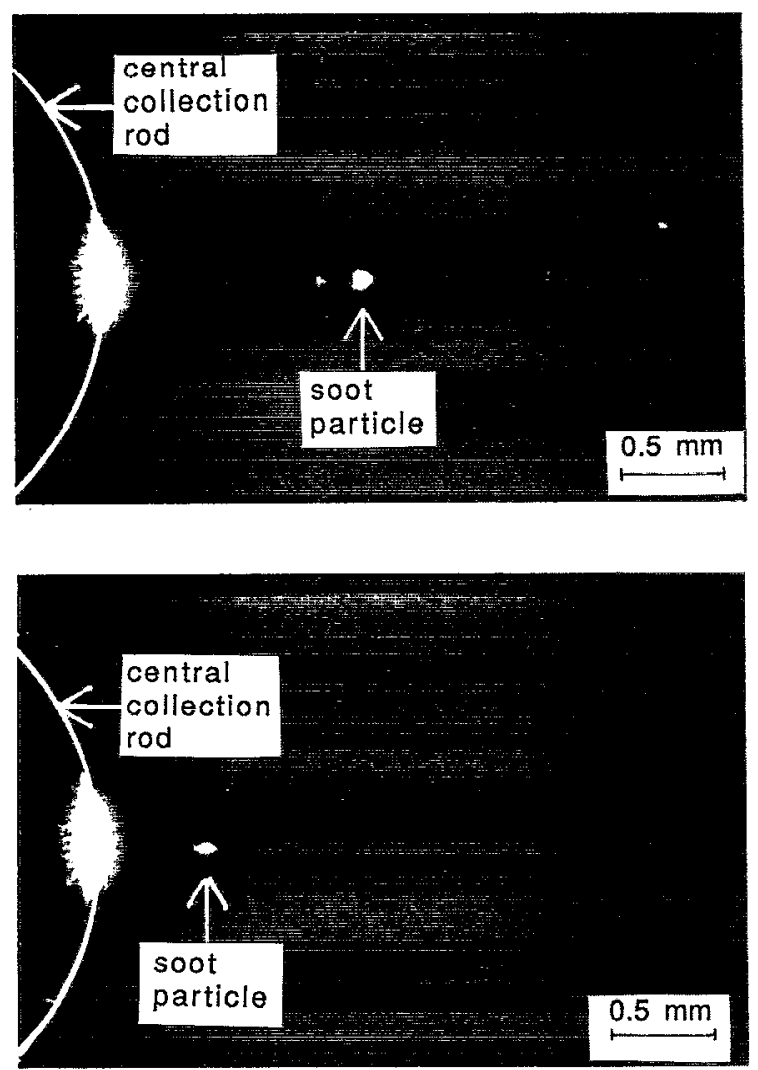

FIG. 10. Photographs showing a particularly slow particle (chosen for photographic clarity) near the collection rod (end view) (top) $0 \mathrm{~s}$, (bottom) 0.17 s. The particle was moving toward the rod with an average speed of $4.5 \mathrm{~mm} / \mathrm{s}$.
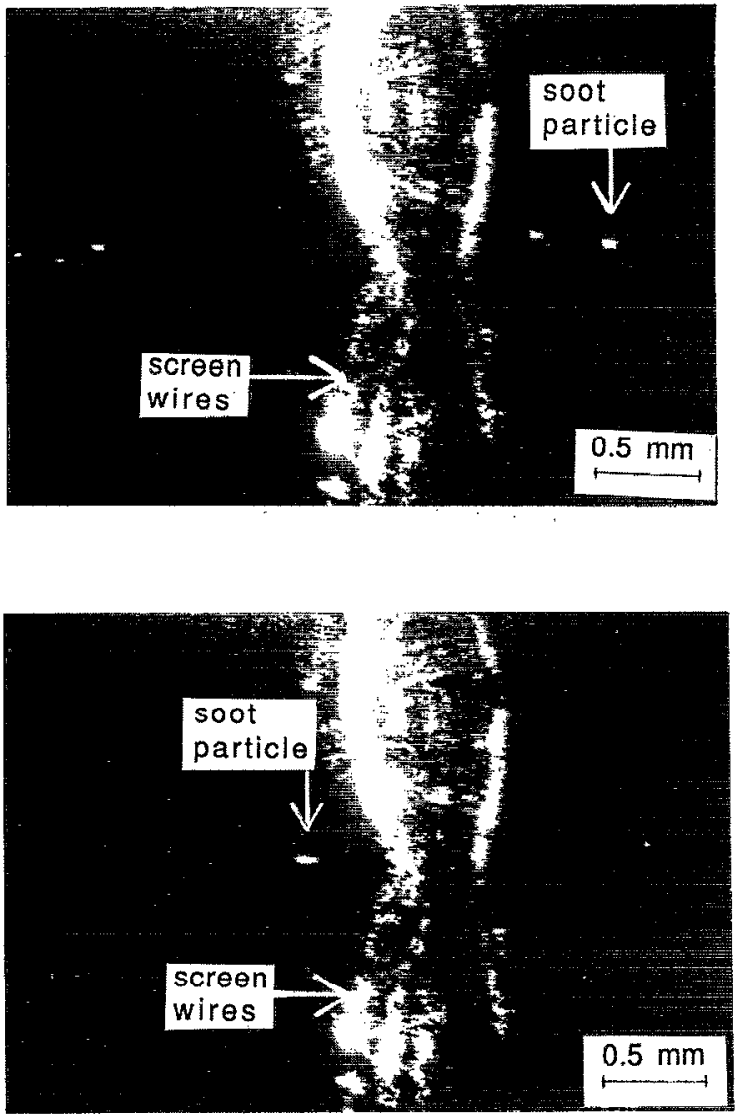

FIG. 11. Photographs showing a particularly slow particle (chosen for photographic clarity) near the screen (end view) (top) $0 \mathrm{~s}$ (bottom) $1.00 \mathrm{~s}$. The particle was charged and moving toward the screen and collection rod to the left (not shown) with an average speed of $1.5 \mathrm{~mm} / \mathrm{s}$.

by Eq. (2) due to its initial location in the chamber and anomalies in the spatial density of ions generated in the charging process. Other particles seen on the video but not photographed had speeds averaging $30 \mathrm{~mm} / \mathrm{s}$-roughly corresponding to the theoretical value of $43 \mathrm{~mm} / \mathrm{s}$. A statistical study of particle migration velocities was not made but would indicate the particle size and charge distributions, and will be attempted in the future.

Motion of particles through the electrified screen was also observed. Figure 11 shows photographs of a slow particle taken at $1.0 \mathrm{~s}$ intervals as the particle passed through the screen. The average speed of this particle was $1.5 \mathrm{~mm} / \mathrm{s}$. Again, the theoretically predicted value is higher than this by one order of magnitude, the difference being mainly due to charge anomalies. Other particles seen on the video but not photographed had speeds of the order of the theoretically predicted values.

\section{DISCUSSION}

The basic design concepts of the inward electrostatic precipitator have been described. The instrument is designed to collect IDPs with diameters ranging from approximately 1 to $1000 \mu \mathrm{m}$ initially dispersed throughout a large volume filled with xenon gas onto a planchet of small size so that the particles can be easily located for inspection and analysis. Particles below $1 \mu \mathrm{m}$ are difficult to charge by field charging 
and particles above $1000 \mu \mathrm{m}$ diam may be too large to move through the screens. (The calculations are valid for particles ranging from about 0.5 to $5 \mu \mathrm{m}$ in radius.) The analysis of the motion of charged particles in an electric field was used to optimize the positions of one, two, or three intermediate screens which greatly enhance the collection speed. Three internal screens appropriately placed in a spherical inward precipitator decrease the collection time by a factor of 185 . The collection time is inversely proportional to the particle size and dielectric constant. For example, a $10-\mu \mathrm{m}$-diam mineral particle $(\kappa \sim 2)$ requires $0.78 \mathrm{~h}$ and a conducting particle $(\kappa \sim 10)$ requires only $0.47 \mathrm{~h}$, while a $1-\mu \mathrm{m}$-diam particle requires 7.8 and $4.7 \mathrm{~h}$, respectively.

The screens also decrease the collection time in a cylindrical precipitator and results are presented for this geometry as well. A cylindrical precipitator with three screens can collect a particle in $67 \%$ of the time required for a spherical precipitator with three screens and with the same overall radius.

Because of the nature of the potential field around the wires in the screens, some particle loss will occur due to impingement. This effect is demonstrated with the use of a finite element solution of Laplace's equation which governs the potential field around an infinite array of wires between two parallel plates in the absence of space charge. The loss was $40 \%$ in this example in which the wire-to-wire spacing was ten times the wire diameter. Losses could be reduced by using a wide spacing between wires in the array.
A cylindrical inward precipitator was constructed for demonstration purposes. A long range microscope, $C C D$ camera and video casette recorder showed $\sim 1 \mu \mathrm{m}$ diameter particles moving through the screen and impinging on the central planchet at speeds of a approximately $30 \mathrm{~mm} / \mathrm{s}$, roughly equivalent to the value of $43 \mathrm{~mm} / \mathrm{s}$ predicted by the theory.

\section{ACKNOWLEDGMENTS}

This research was supported under NASA Grant Nos. NAG-1953 and NAGW-1941 and National Science Foundation Grant No. CBT8813006. Contribution No. 5077, Division of Geological and Planetary Science.

${ }^{2}$ E. Grūn, H. A. Zook, H. Fechtig, and R. H. Giese, Icarus 62, 244 (1985).

${ }^{2}$ N. Divine, J. Geophys. Res. 98, 17029 (1993).

${ }^{3}$ P. Tsou, Int. J. Impact Eng. 10, 615 (1990)

${ }^{4}$ A. J. Rulison, R. C. Flagan, T. J. Ahrens, and W. F. Mịller, Astrophys. J. 371, 432 (1991)

${ }^{5}$ J. P. Bradley and D. E. Brownlee, Science 251, 549 (1991).

${ }^{5} \mathrm{E}$. C. M. Chen and W. E. Wentworth, J. Chem. Ed. 52, 486 (1975).

${ }^{7}$ R. C. Flagan and J. H. Seinfeld, Fundamentals of Air Pollution Engineering (Prentice-Hall, Englewood Cliffs, 1988).

${ }^{8}$ W. H. Press, B. P. Flannery, S. A. Teukolsky, and W. T. Vetterling, $\mathrm{Nu}$ merical Recipes, the Art of Scientific Computing (FORTRAN Version) (Cambridge University Press, Cambridge, 1989).

${ }^{9}$ D. A. Anderson, J. C. Tannehill, and R. H. Pletcher, Computational Fluid Mechanics and Heat Transfer (McGraw-Hill, Washington, 1984).

${ }^{10}$ B. S. Hayes and H. G. Wagner, Prog. Energy Combust. Sci. 7, 229 (1981). 